REVISTA MATEMÁTICA COMPLUTENSE

Volumen 12, número 1: 1999

http://dx.doi.org/10.5209/rev_REMA.1999.v12.n1.17191

\title{
The density condition in quotients of quasinormable Fréchet spaces, II.
}

\author{
Angela A. ALBANESE
}

\begin{abstract}
It is proved that a Frechet space is quasinormable if, and only if, every quotient space satisfies the density condition of Heinrich. This answers positively a conjecture of Bonet and Díaz.
\end{abstract}

The class of quasinormable locally convex spaces was introduced and studied by Grothendieck in [10]. This class has recently received much attention in the context of Fréchet spaces and of Köthe sequence spaces (see $[5,6,7,8,9,15,16]$ ). In particular Bonet, Díaz $[7,8]$ and Díaz, Fernández [9] proved that a Köthe sequence space of order $p, 1 \leq p \leq \infty$ or $p=0$, is quasinormable if, and only if, every quotient space satisfies the density condition of Heinrich [11]. This result is related to important previous theorems by Bellenot [3] and Valdivia [17], namely, a Fréchet space is Schwartz (respectively, totally reflexive) if every quotient is Montel (respectively, reflexive). Accordingly, the following question was asked as Open Problem 15 in [2]: If every quotient of a Fréchet space $E$ has the density condition, is $E$ quasinormable? In [1] we showed that the answer is positive under the assumption that $E$ is separable. In this note we remove this hypothesis hence we provide a complete solution to the just mentioned problem.

In what follows we recall some notation.

In the sequel, given a Fréchet space $E$ we denote by $\left(\|\|_{k}\right)_{k}$ an increasing fundamental system of seminorms defining the topology of $E$ such that the sets

1991 Mathematics Subject Classification: 46A04.

Research partially supported by the Italian MURST.

Servicio Publicaciones Univ. Complutense. Madrid, 1999. 
$U_{k}:=\left\{x \in E ;\|x\|_{k} \leq 1\right\}$ form a basis of 0 -neighbourhoods in $E$. The dual seminorms are defined by

$$
\|f\|_{k}^{\prime}:=\sup \left\{|f(x)| ;\|x\|_{k} \leq 1\right\}=\sup \left\{|f(x)| ;\|x\|_{k}=1\right\}
$$

for $f \in E^{\prime}$; hence \|\|$_{k}^{\prime}$ is the gauge of $\stackrel{0}{U}_{k}$ in $E^{\prime}$. We denote by $E_{k}^{\prime}:=\left\{f \in E^{\prime} ;\|f\|_{k}^{\prime}<\infty\right\}$ the linear span of $\stackrel{\circ}{U}_{k}$ endowed with the norm topology defined by \|\|$_{k}^{\prime}$. Clearly, $\left(E_{k}^{\prime},\|\|_{k}^{\prime}\right)$ is a Banach space and $E_{k}^{\prime}=\left(\frac{E}{k e r\|\|_{k}}, \Perp_{k}\right)^{\prime}$

We always mean separated quotient spaces for quotient spaces.

A Fréchet space $E$ is called quasinormable if there exists a bounded subset $B$ of $E$ such that

$$
\forall n \exists m>n \forall \varepsilon>0 \exists \lambda>0 \text { : } U_{m} \subset \lambda B+\varepsilon U_{n} .
$$

By $[15$, Theorem 7] (cf. [6, Theorem]) a Fréchet space $E$ is quasinormable if, and only if,

$$
\forall n \exists m>n \forall k>m \forall \varepsilon>0 \exists \lambda>0 \quad: \quad U_{m} \subset \lambda U_{k}+\varepsilon U_{n} . \quad(Q N)_{1}
$$

By polarization it follows that a Fréchet space $E$ is quasinormable if, and only if,

$$
\forall n \exists m>n \forall k>m \forall \varepsilon>0 \exists \lambda>0 \quad: \lambda \stackrel{\circ}{U}_{k} \cap \stackrel{\circ}{U}_{n} \subset \varepsilon{\stackrel{\circ}{U_{m}}}^{\prime} \quad(Q N)_{2}
$$

The density condition was introduced by Heinrich in his study of ultrapowers of locally convex spaces [11]. A Fréchet space $E$ is called to satisfy the density condition (see [4, Proposition 2]) if for any sequence $\left(\lambda_{n}\right)_{n}$ of strictly positive numbers there exists a bounded subset $B$ of $E$ such that

$$
\forall n \exists m>n \exists \lambda>0: \bigcap_{j=1}^{m} \lambda_{j} U_{j} \subset \lambda B+U_{n} .
$$

The density condition was thoroughly studied for Fréchet and Köthe spaces by Bieisiedt and Bonet [4]. It was proved there that a Fréchet space $E$ has the density condition if, and only if, the bounded subsets of its strong dual are metrizable. Every quasinormable and every Montel Fréchet space has the density condition. 
We also recall the following fact due to Díaz and Fernández [9, Lemma 1] which is useful for the sequel:

Lemma 0. Let $E$ be a Fréchet space. Suppose that there exist sequences $\left(x_{j k}\right)_{j, k \in \mathrm{N}} \subset E$ and $\left(f_{j k}\right)_{j, k \in \mathrm{N}} \subset E^{\prime}$ with the following properties:

(1) $f_{j k}\left(x_{j k}\right)=1, \forall j, k \in \mathrm{N}$,

(2) $\left\|f_{j k}\right\|_{1}^{\prime} \leq 1, \forall j, k \in \mathbf{N}$,

(3) $M_{k}=\sup \left\{\left\|x_{j n}\right\|_{k} ; j \in \mathbf{N}, n \geq k\right\}<+\infty, \forall k \in \mathbf{N}$,

(4) $\lim _{j \rightarrow+\infty}\left\|f_{j k}\right\|_{k+1}^{\prime}=0, \forall k \in \mathbf{N}$.

Then $E$ does not satisfy the density condition of Heinrich.

Let $E$ be a Fréchet space. Let $\left(x_{n}\right)_{n \in \mathbf{N}} \subset E$ and $\left(f_{n}\right)_{n \in \mathbb{N}} \subset E^{\prime}$. The couple of sequences $\left(x_{n}, f_{n}\right)_{n \in \mathbb{N}}$ is called a biorthogonal system if $f_{n}\left(x_{m}\right)=\delta_{n m}$ for every $n$ and $m \in \mathbf{N}$. If \|\| is a seminorm of $E$, a sequence $\left(x_{n}\right)_{n \in \mathbb{N}}$ is called a basic sequence with respect to \|\| if there exists a positive constant $K$ such that for all scalars $\left(\alpha_{n}\right)_{n}$ and $p$ and $q$ in $\mathbf{N}$

$$
\left\|\sum_{n=1}^{p} \alpha_{n} x_{n}\right\| \leq K\left\|\sum_{n=1}^{p+q} \alpha_{n} x_{n}\right\| .
$$

For all undefined notation we refer to $[12,13]$.

\section{§1. The result.}

In order to state and to prove our result we need the following two lemmas. The first one provides conditions to ensure that a Fréchet space has a quotient space which does not satisfy the density condition. Its proof is based on Lemma 0 and on an useful method due to Bonet and Díaz for constructing quotient spaces without the density condition in the setting of Köthe sequence spaces, see $[8$, Sect. 3 , Proposition 2] or [7, Theorem 3].

Lemma 1. Let $E$ be a Fréchet space with a continuous norm. Suppose that there exist sequences $\left(x_{j k}\right)_{j, k \in \mathbb{N}} \subset E$ and $\left(f_{j k}\right)_{j, k \in \mathbb{N}} \subset E^{\prime}$ with the following properties:

(a) $\left(x_{j k}, f_{j k}\right)_{j, k \in \mathbf{N}}$ is a biorthogonal system, 
(b) $\left\|f_{j k}\right\|_{1}^{\prime} \leq 1, \forall j, k \in \mathbf{N}$,

(c) $\sup \left\{\left\|x_{j k}\right\|_{k} ; j \in \mathbf{N}\right\}<+\infty, \forall k \in \mathbf{N}$,

(d) $\lim _{j \rightarrow+\infty}\left\|f_{j k}\right\|_{k+1}^{\prime}=0, \forall k \in \mathbf{N}$.

Then $E$ has a quotient space which does not satisfy the density condition of Heinrich.

Proof. We split the first $\mathbf{N}$ in $\mathbf{N} \times \mathbf{N}$ as a countable union of disjoint infinite subsets, hence we may write $\left(x_{i j k}, f_{i j k}\right)_{i, j, k \in \mathbf{N}}$ instead of $\left(x_{j k}, f_{j k}\right)_{j, k \in \mathbb{N}}$. Thus we obtain:

$$
\begin{gathered}
\left\|f_{i j k}\right\|_{1}^{\prime} \leq 1, \quad \forall i, j \text { and } k \in \mathbf{N}, \\
\sup \left\{\left\|x_{i j k}\right\|_{n}: i, j \in \mathbf{N}\right\}=\alpha_{n k}<+\infty, \forall k \in \mathbf{N}, \forall n \leq k, \\
\lim _{i \rightarrow+\infty}\left\|f_{i j k}\right\|_{k+1}^{\prime}=0, \forall j, k \in \mathbf{N} .
\end{gathered}
$$

Now, we put

$$
c(i, j, k)=\max \left\{\left\|f_{i j s}\right\|_{j+1}^{\prime}: 1 \leq s \leq j\right\} \text { if } j<k
$$

and

We define

$$
c(i, j, k)=1 \text { if } 1 \leq k \leq j
$$

$$
g_{i j}: E \rightarrow \mathbf{R}, x \rightarrow \sum_{k=1}^{\infty} f_{i j k}(x) \frac{c(i, j, k)}{2^{k}}
$$

By (1), we have that, for each $i, j \in \mathbf{N}$,

$$
\left|g_{i j}(x)\right| \leq\|x\|_{1} \sum_{k=1}^{\infty}\left\|f_{i j k}\right\|_{1}^{\prime} 2^{-k} \leq\|x\|_{1} ;
$$

hence

$$
\left\|g_{i j}\right\|_{1}^{r} \leq 1
$$

By (1) again, we obtain

$$
\begin{aligned}
\mid g_{i j}(x) & \leq\left(\sum_{k=1}^{j}\left\|f_{i j k}\right\|_{j+1}^{\prime} \frac{1}{2^{k}}+\sum_{k>j}\left\|f_{i j k}\right\|_{j+1}^{\prime} \frac{c\left(\frac{i, j, k)}{2^{k}}\right)}{\|x\|_{j+1}}\right. \\
& \leq \max \left\{\left\|f_{i j k}\right\|_{j+1}^{\prime} ; 1 \leq k \leq j\right\}\|x\|_{j+1}
\end{aligned}
$$


hence $\left\|g_{i j}\right\|_{j+1}^{\prime} \leq \max \left\{\left\|f_{i j k}\right\|_{j+1}^{\prime} ; 1 \leq k \leq j\right\} \leq \max \left\{\left\|f_{i j k}\right\|_{k+1}^{\prime} ; 1 \leq\right.$ $k \leq j\}$ and so, by (3),

$$
\lim _{i \rightarrow+\infty}\left\|g_{i j}\right\|_{j+1}^{\prime}=0
$$

Now we are ready to construct the desired quotient space.

Let $F=\cap_{i, j \in N}$ ker $g_{i j}$. We consider the quotient space $E / F$, with quotient map $T: E \rightarrow E / F$, and we check that it does not satisfies the density condition. To do this it suffices to prove that $E / F$ satisfies all conditions of Lemma 0 .

We denote by $\left(\|\| \mid\|\|_{k}\right)_{k}$ the quotient norms induced by $\left(\|\|_{k}\right)_{k}$ on $E / F$ (each \|\|\|\|$_{k}$ is a norm because $F$ is closed with respect to every \|\|$_{k}$ ) and put, for each $i, j \in \mathbf{N}$ and $x \in E$,

$$
\bar{g}_{i j}(T x)=g_{i j}(x) .
$$

Clearly, $\bar{g}_{i j}$ is well-defined, linear and continuous. Further, since

$$
\left\|\left|\bar{g}_{i j}\right|\right\|_{k}^{\prime}=\sup _{\|T x\| \|_{k}<1}\left|\bar{g}_{i j}(T x)\right|=\sup _{\|x\|_{k}<1}|g(x)|=\left\|g_{i j}\right\|_{k}^{\prime}
$$

for every $i, j$ and $k \in \mathbf{N}$, by (4) and (5) it follows that

$$
\left\|\left|\bar{g}_{i j}\right|\right\|_{1}^{\prime} \leq 1 \text { and } \lim _{i \rightarrow+\infty}\left\|\bar{g}_{i j} \mid\right\|_{j+1}^{\prime}=0 \text {. }
$$

Next, put $z_{i j}=T\left(2 x_{i j 1}\right)$ for every $i, j \in \mathrm{N}$. Then, by (a) $\bar{g}_{i j}\left(z_{l s}\right)=$ $\delta_{i l} \delta_{j s}$, thereby implying that $\left(z_{i j}, \bar{g}_{i j}\right)_{i, j} \in E / F \times(E / F)^{\prime}$ is a biorthogonal system.

Finally, fix $j \in \mathbf{N}$ and given $n \geq j$, we have $2 x_{i n 1}-2^{j} x_{i n j} \in F$ and hence, by (2),

$$
\left\|\mid z_{i n}\right\|\left\|_{j} \leq\right\| 2 x_{i n 1}-\left(2 x_{i n 1}-2^{j} x_{i n j}\right)\left\|_{j} \leq 2^{j}\right\| x_{i n j} \|_{j} \leq 2^{j} \alpha_{j j}
$$

it follows that, for each $j \in \mathbf{N}$,

$$
M_{j}=\sup \left\{\|\| z_{i n} \|_{j} ; i \in \mathbf{N}, n \geq j\right\}<+\infty .
$$

We have so proved that the biorthogonal system $\left(z_{i j}, \bar{g}_{i j}\right)_{i, j} \subset E / F \times$ $(E / F)^{\prime}$ satisfies all conditions $(1) \div(4)$ of Lemma 0 . Therefore, Lemma 
0 can be applied to conclude that $E / F$ does not satisfy the density condition and the result follows.

The next lemma is the second basic step towards our result. It provides the main technical tool for constructing a biothogonal system satisfying all the above conditions (a) $\div$ (d) in a given Fréchet space which is not quasinormable without the assumption of the separability:

Lemma 2. Let $E$ be a Fréchet space. Let $G$ be a subspace of $E^{\prime}$ so that

$$
\inf \left\{\|f\|_{k+1}^{\prime} ; f \in G,\|f\|_{k}^{\prime}>\beta,\|f\|_{1}^{\prime}<\alpha\right\}=0
$$

for some $k \in \mathbf{N}$ and $\alpha, \beta>0$.

Then, if $B$ is a finite-dimensional subspace of $E_{k}^{\prime}$ and $\varepsilon, \mu>0$, there exists an $f \in G$ with $\|f\|_{1}^{\prime}<\alpha,\|f\|_{k}^{\prime}>\beta$ and $\|f\|_{k+1}^{\prime}<\mu$ so that

$$
\|g\|_{k}^{\prime} \leq(1+\varepsilon)\|g+\lambda f\|_{k}^{\prime},
$$

for every $g \in B$ and every scalar $\lambda$.

Proof. By assumption we can find a sequence $\left(f_{j}\right)_{j \in \mathbf{N}} \subset G$ so that $\left\|f_{j}\right\|_{1}^{\prime}<\alpha,\left\|f_{j}\right\|_{k}^{\prime}>\beta$ for every $j \in \mathbf{N}$ and $\lim _{j \rightarrow \infty}\left\|f_{j}\right\|_{k+1}^{\prime}=0$. It follows that $\left(f_{j}\right)_{j \in \mathbf{N}}$ converges to 0 in $E_{\beta}^{\prime}$ and hence it converges to 0 in $E_{\sigma}^{\prime}=\left(E^{\prime}, \sigma\left(E^{\prime}, E\right)\right)$. We shall show that there is an element of $\left(f_{j}\right)_{j \in \mathbf{N}}$ with the desired properties. To do this we proceed in a similar way as in [14, Lemma 1.a.6].

We may clearly assume $\varepsilon<1$. Put $\varepsilon^{\prime}=\varepsilon / 2$. Let $\left(g_{i}\right)_{i=1}^{m} \subset B$ with $\left\|g_{i}\right\|_{k}^{\prime}=1$ such that for every $g \in B$ with $\|g\|_{k}^{\prime}=1$ there is an $i \in\{1, \ldots, m\}$ for which $\left\|g-g_{i}\right\|_{k}^{\prime}<\varepsilon^{\prime} / 4$. By definition of \|\|$_{k}^{\prime}$, there exists $\left(x_{i}\right)_{i=1}^{m} \subset E$ such that $\left\|x_{i}\right\|_{k}=1$ and $1-\varepsilon^{\prime} / 4<g_{i}\left(x_{i}\right) \leq 1$.

Since $\left(f_{j}\right)_{j \in \mathbf{N}}$ converges to 0 in $E_{\sigma}^{\prime}$ and $\lim _{j \rightarrow \infty}\left\|f_{j}\right\|_{k+1}^{\prime}=0$, we can choose a $j_{0} \in \mathbf{N}$ large enough to have $\left|f_{j_{0}}\left(x_{i}\right)\right|<\varepsilon^{\prime} \beta / 4$ for every $i=1, \ldots, m$ and $\left\|f_{j_{0}}\right\|_{k+1}^{\prime}<\mu$. We now put $f_{0}=f_{j_{0}} /\left\|f_{j_{0}}\right\|_{k}^{\prime}$. Then $\left\|f_{0}\right\|_{k}^{\prime}=1$ and $\left|f_{0}\left(x_{i}\right)\right|<\varepsilon^{\prime} / 4$ for every $i=1, \ldots, m$.

Now, if $g \in B$ with $\|g\|_{k}^{\prime}=1$ and $|\lambda| \geq 2$, then

$$
\left\|g+\lambda f_{0}\right\|_{k}^{\prime} \geq|\lambda|\left\|f_{0}\right\|_{k}^{\prime}-\|g\|_{k}^{\prime} \geq 1=\|g\|_{k}^{\prime} .
$$

If $|\lambda|<2$, we take $i$ such that $\left\|g-g_{i}\right\|_{k}^{\prime}<\varepsilon^{\prime} / 4$ and then

$$
\begin{aligned}
\left\|g+\lambda f_{0}\right\|_{k}^{\prime} & \geq\left\|g_{i}+\lambda f_{0}\right\|_{k}^{\prime}-\left\|g-g_{i}\right\|_{k}^{\prime} \geq\left(g_{i}+\lambda f_{0}\right)\left(x_{i}\right)-\varepsilon^{\prime} / 4 \\
& >\left(1-\varepsilon^{\prime} / 4\right)-\varepsilon^{\prime} / 2-\varepsilon^{\prime} / 4=\left(1-\varepsilon^{\prime}\right)\|g\|_{k}^{\prime} .
\end{aligned}
$$


Therefore we have that

$$
\|g\|_{k}^{\prime} \leq \frac{1}{1-\varepsilon^{\prime}}\left\|g+\lambda f_{0}\right\|_{k}^{\prime} \leq(1+\varepsilon)\left\|g+\lambda f_{0}\right\|_{k}^{\prime}
$$

for every $g \in B$ and every scalar $\lambda$. Recalling that $f_{j_{0}}=\left\|f_{j_{0}}\right\|_{k}^{\prime} f_{0}$, the result follows.

We have now our main result which should be compared with Theorem 4 of [1].

Theorem 3. A Fréchet space $E$ is quasinormable if, and only if, every quotient space satisfies the density condition of Heinrich.

Proof. Every quotient space of a quasinormable space is again quasinormable and hence has the density condition. Thus the necessity of the condition follows.

We now suppose that $E$ is non-quasinormable Fréchet space. Our aim is to construct a biorthogonal sequence which satisfies the hypotheses of Lemma 1, thus $E$ has a quotient space without the density condition and that finishes the proof. By Lemma 3 of [1] we may assume that $E$ has a continuous norm. Let (\|l $\left.\|_{k}\right)_{k \in \mathbb{N}}$ be an increasing fundamental sequence of norms for $E$.

Since $E$ is non quasinormable, by using $(Q N)_{2}$, we have

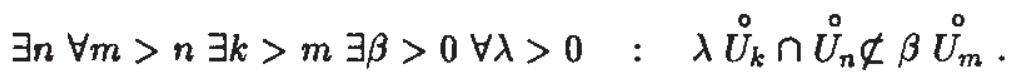

Without loss of generality, we may then assume that there is a decreasing sequence $\left(\beta_{k}\right)_{k \in N}$ of numbers with $0<\beta_{k}<1$ so that

$$
\forall k \in \mathbf{N} \forall \lambda>0 \quad ; \quad \lambda \stackrel{\circ}{U}_{k+1} \cap \stackrel{\circ}{U}_{1} \not \subset \beta_{k} \stackrel{\circ}{U}_{k},
$$

or equivalently

$$
\forall k \in \mathbf{N} \quad \inf \left\{\|f\|_{k+1}^{\prime} ; f \in E^{\prime},\|f\|_{1}^{\prime} \leq 1,\|f\|_{k}^{\prime}>\beta_{k}\right\}=0 .
$$

Actually, we have more. If $G=\left\{f \in E^{\prime} ; f(x)=0 \forall x \in L\right\}$ with $L$ some finite subset of $E$, from (7) it follows that, for each $k \in \mathbf{N}$ and $0<\delta<\beta_{k}$,

$$
\inf \left\{\|f\|_{k+1}^{\prime} ; f \in G,\|f\|_{1}^{\prime}<1+\delta,\|f\|_{k}^{\prime}>\beta_{k}-\delta\right\}=0 .
$$


The proof of this fact is contained in [1, Theorem 1$]$ and we repeat it here for the sake of completeness.

Assume that $L=\left(z_{i}\right)_{i=1}^{m}$ is a linearly independent set of $E$. Since \|\|$_{1}$ is a norm on $E, E_{1}^{\prime}$ is $\sigma\left(E^{\prime}, E\right)$-dense in $E^{\prime}$ so we can find $\left(g_{i}\right)_{i=1}^{m} \subset E_{1}^{\prime}$ such that $\left(z_{i}, g_{i}\right)_{i=1}^{m}$ is biorthogonal. Then the map $P: E^{\prime} \rightarrow E^{\prime}$ defined by $P f=\sum_{i=1}^{m} f\left(z_{i}\right) g_{i}$ is a projection with $\operatorname{ker} P=G$ and $P$ is continuous with respect to each norm \|\|$_{k}^{r}$.

Now, fix $k \in \mathbf{N}$ and $0<\delta<\beta_{k}$. By (7) we can find a sequence $\left(f_{j}\right)_{j} \subset E_{1}^{\prime}$ with $\left\|f_{j}\right\|_{1}^{\prime} \leq 1,\left\|f_{j}\right\|_{k}^{\prime}>\beta_{k}$ for each $j \in \mathbf{N}$ and $\lim _{j \rightarrow \infty}\left\|f_{j}\right\|_{k+1}^{\prime}=0$. Then $\lim _{j \rightarrow \infty}\left\|P f_{j}\right\|_{k+1}^{\prime}=0$ too; hence, since $P\left(E_{1}^{\prime}\right)$ is finite dimensional, also $\lim _{j \rightarrow \infty}\left\|P f_{j}\right\|_{1}^{\prime}=0$. Therefore, there exists a $j_{0} \in \mathbf{N}$ such that $\left\|P f_{j}\right\|_{1}^{\prime}<\delta$ for each $j \geq j_{0}$. It follows that the sequence $\left(h_{j}\right)_{j \geq j_{0}}=\left(f_{j}-P f_{j}\right)_{j \geq j_{0}} \subset G$ satisfies the following

$$
\left\|h_{j}\right\|_{1}^{\prime} \leq\left\|f_{j}\right\|_{1}^{\prime}+\left\|P f_{j}\right\|_{1}^{\prime}<1+\delta
$$

and

$$
\left\|h_{j}\right\|_{k}^{\prime} \geq\left\|f_{j}\right\|_{k}^{\prime}-\left\|P f_{j}\right\|_{k}^{\prime}>\beta_{k}-\delta
$$

for each $j \geq j_{0}$. Clearly, also $\lim _{j \rightarrow \infty}\left\|h_{j}\right\|_{k+1}^{\prime}=0$. We may then conclude that assertion (8) holds.

We now construct by induction a biorthogonal system in $E \times E^{\prime}$ satisfying all conditions of Lemma 1.

Let $\gamma: \mathbf{N} \rightarrow \mathbf{N}^{2}$ be a bijective map and put $\gamma(n)=\left(\gamma_{1}(n), \gamma_{2}(n)\right)$.

For $i=1$ we take $\gamma_{2}(1)$; by (7) we find $f_{\gamma(1)} \in E^{\prime}$ with

$$
\left\|f_{\gamma(1)}\right\|_{1}^{\prime} \leq 1,\left\|f_{\gamma(1)}\right\|_{\gamma_{2}(1)}^{\prime}>\beta_{\gamma_{2}(1)} \text { and }\left\|f_{\gamma(1)}\right\|_{\gamma_{2}(1)+1}^{\prime}<\gamma_{2}(1)^{-\gamma_{1}(1)} \text {. }
$$

Since $\beta_{\gamma_{2}(1)}<\left\|f_{\gamma(1)}\right\|_{\gamma_{2}(1)}^{\prime} \leq 1$, there is an $x_{\gamma(1)} \in E$ with $\left\|x_{\gamma(1)}\right\|_{\gamma_{2}(1)}<$ $\left(\beta_{\gamma_{2}(1)}\right)^{-1}$ and $f_{\gamma(1)}\left(x_{\gamma(1)}\right)=1$.

Assume we have determined a biorthogonal system $\left(x_{\gamma(i)}, f_{\gamma(i)}\right)_{i=1}^{n}$ such that, for each $i=1, \ldots, n$,

$$
\begin{gathered}
\left\|f_{\gamma(i)}\right\|_{\gamma_{2}(i)+1}^{\prime}<\gamma_{2}(i)^{-\gamma_{1}(i)}, \frac{\beta_{\gamma_{2}(i)}}{2}<\left\|f_{\gamma^{(i)}}\right\|_{\gamma_{2}(i)}^{\prime} \leq\left\|f_{\gamma(i)}\right\|_{1}^{\prime}<2, \\
\left\|x_{\gamma^{\prime}(i)}\right\|_{\gamma_{2}(i)}<\frac{6}{\beta_{\gamma_{2}(i)}}
\end{gathered}
$$


and

$$
\|g\|_{\gamma_{2}(i)}^{\prime} \leq\left(1+\varepsilon_{i}\right)\left\|g+\lambda f_{\gamma(i)}\right\|_{\gamma_{2}(i)}^{\prime}
$$

for every $g \in B_{i-1}=\left[f_{\gamma(j)} ; j=1, \ldots, i-1\right]$ and for every scalar $\lambda$, where $0<\varepsilon_{i}<1$ for $i=1, \ldots, n$.

Then, consider the space

$$
G_{n+1}=\left\{f \in E^{\prime} ; f\left(x_{\gamma(i)}\right)=0 \text { for } i=1, \ldots, n\right\} .
$$

For $i=n+1$ we take $\gamma_{2}(n+1)$ and $\delta=\frac{\overline{\beta_{\gamma_{2}(n+1)}}}{2}$, then, by (8) we have

$$
\begin{aligned}
& \inf \left\{\|f\|_{\gamma_{2}(n+1)+1}^{\prime} ; f \in G_{n+1},\|f\|_{1}^{\prime}<1+\frac{\beta_{\gamma_{2}(n+1)}}{2}\right. \\
&\left.\|f\|_{\gamma_{2}(n+1)}^{\prime}>\frac{\beta_{\gamma_{2}(n+1)}}{2}\right\}=0 .
\end{aligned}
$$

Since $B_{n}=\left[f_{\gamma(i)} ; i=1, \ldots, n\right]$ is a finite dimensional subspace of $E_{1}^{\prime}$ and hence of $E_{\gamma_{2}(n+1)}^{\prime}$, taken $0<\varepsilon_{n+1}<1$ and $\mu=\gamma_{2}(n+1)^{-\gamma_{1}(n+1)}$, by Lemma 2 , there exists an $f_{\gamma(n+1)} \in G_{n+1}$ such that

$$
\begin{gathered}
\left\|f_{\gamma(n+1)}\right\|_{\gamma_{2}(n+1)+1}^{\prime}<\gamma_{2}(n+1)^{-\gamma_{1}(n+1)} \\
\frac{\beta_{\gamma_{2}(n+1)}}{2}<\left\|f_{\gamma(n+1)}\right\|_{\gamma_{2}(n+1)}^{\prime} \leq\left\|f_{\gamma(n+1)}\right\|_{1}^{\prime}<1+\frac{\beta_{\gamma_{2}(n+1)}}{2}<2
\end{gathered}
$$

and

$$
\|g\|_{\gamma_{2}(n+1)}^{\prime} \leq\left(1+\varepsilon_{n+1}\right)\left\|g+\lambda f_{\gamma(n+1)}\right\|_{\gamma_{2}(n+1)}^{\prime}
$$

for every $g \in B_{n}$ and for every scalar $\lambda$.

If $g_{n+1}^{\prime} \in\left[f_{\gamma(i)} ; i=1, \ldots, n+1\right]^{\prime}$ is defined by $g_{n+1}^{\prime}\left(\sum_{i=1}^{n+1} a_{i} f_{\gamma(i)}\right)=$ $a_{n+1}$ (i.e., $g_{n+1}^{\prime} \in E_{\gamma_{2}(n+1)}^{\prime \prime}$, where $E_{\gamma_{2}(n+1)}^{\prime \prime}$ is the strong bidual of the normed space $\left.E_{\gamma_{2}(n+1)}=\left(E,\|\|_{\gamma_{2}(n+1)}\right)\right)$, then, by (9) we get

$$
\left|g_{n+1}^{\prime}(g)\right|=\left|g_{n+1}^{\prime}\left(\sum_{i=1}^{n+1} a_{i} f_{\gamma(i)}\right)\right|=\frac{\left\|a_{n+1} f_{\gamma(n+1)}\right\|_{\gamma_{2}(n+1)}^{\prime}}{\left\|f_{\gamma(n+1)}\right\|_{\gamma_{2}(n+1)}^{\prime}}
$$




$$
\begin{aligned}
& <\frac{2}{\beta_{\gamma_{2}(n+1)}}\left\|g-\sum_{i=1}^{n} a_{i} f_{\gamma^{(i)}}\right\|_{\gamma_{2}(n+1)}^{\prime} \\
& \leq \frac{2}{\beta_{\gamma_{2}(n+1)}}\left(\|g\|_{\gamma_{2}(n+1)}^{\prime}+\left\|\sum_{i=1}^{n} a_{i} f_{\gamma(i)}\right\|_{\gamma_{2}(n+1)}^{\prime}\right) \\
& \leq \frac{2}{\beta_{\gamma_{2}(n+1)}}\left(2+\varepsilon_{n+1}\right)\|g\|_{\gamma_{2}(n+1)}^{\prime}
\end{aligned}
$$

therefore $\left\|g_{n+1}^{\prime}\right\|_{\gamma_{2}(n+1)}^{\prime}<\frac{6}{\beta_{\gamma_{2}(n+1)} \text {. }}$.

Since $E_{\gamma_{2}(n+1)}^{\prime}$ is the dual of the normed space $E_{\gamma_{2}(n+1)}$, we can then apply Helly's Lemma (see [12, Lemma 8.7 .9$, p. 165]) to find an $x_{\gamma(n+1)} \in$ $E$ so that $\left\|x_{\gamma(n+1)}\right\|_{\gamma_{2}(n+1)}<\frac{6}{\beta_{\gamma_{2}(n+1)}}$ and $f_{\gamma(i)}\left(x_{\gamma(n+1)}\right)=g_{n+1}^{\prime}\left(f_{\gamma(i)}\right)$ for $i=1, \ldots, n+1$ (i.e., $f_{\gamma(n+1)}\left(x_{\gamma(n+1)}\right)=1$ and $f_{\gamma(i)}\left(x_{\gamma(n+1)}\right)=0$ for $i=1, \ldots, n)$.

Proceeding inductively, we construct a biorthogonal system $\left(x_{\gamma(n)}\right.$, $\left.f_{\gamma(n)}\right)_{n} \subset E \times E^{\prime}$ satisfying

$$
\begin{gathered}
\left\|f_{\gamma(n)}\right\|_{1}^{\prime}<2, \quad \forall n \in \mathbf{N}, \\
\left\|f_{\gamma(n)}\right\|_{\gamma_{2}(n)}^{\prime}>\frac{\beta_{\gamma_{2}(n)}}{2}, \forall n \in \mathbf{N}, \\
\left\|f_{\gamma_{(n)}}\right\|_{\gamma_{2}(n)+1}^{\prime}<\gamma_{2}(n)^{-\gamma_{1}(n)}, \forall n \in \mathbf{N}, \\
\left\|x_{\gamma(n)}\right\|_{\gamma_{2}(n)}<\frac{6}{\beta_{\gamma_{2}(n)}}, \forall n \in \mathbf{N}, \\
\|g\|_{\gamma_{2}(n)}^{\prime} \leq \quad\left(1+\varepsilon_{n}\right)\left\|g+\lambda f_{\gamma^{(n)}}\right\|_{\gamma_{2}(n)}^{\prime}, \\
\forall n \in \mathbf{N}, \forall g \in\left[f_{\gamma(i)} ; i=1, \ldots, n-1\right], \forall \lambda,
\end{gathered}
$$

where $\left(\varepsilon_{n}\right)_{n \in \mathbb{N}}$ is any sequence of numbers with $0<\varepsilon_{n}<1$.

Now, put $g_{j k}=\frac{1}{2} f_{j k}$ and $z_{j k}=2 x_{j k}$ for each $j$ and $k \in \mathbf{N}$ (recall that $\left.\gamma(n)=\left(\gamma_{1}(n), \gamma_{2}(n)\right)\right)$, from $(10),(12)$ and (13) follows that the biorthogonal system $\left(z_{j k}, g_{j k}\right)_{j, k \in \mathrm{N}}$ satisfies all conditions $(\mathrm{a}) \div(\mathrm{d})$ of 
Lemma 1 and apply it to conclude that $E$ has a quotient space without the density condition. This finishes the proof.

Note that (14) gives the possibility to choose $\left(g_{j k}\right)$ in such a way that $\left(g_{j k}\right)_{j \in \mathrm{N}}$ is a basic sequence with respect to the dual norm \|\|$_{k}^{t}$ for every $k \in \mathbf{N}$.

\section{References}

[1] A.A. Albanese, The Density Condition in Quotients of Quasinormable Fréchet Spaces, Studia Math. 125 (1997), 131-141.

[2] A. Aytuna, P.B. Djakov, A.P. Goncharov, T. Terzioğlu, V. P. Zahariuta, Some Open Problems in the Theory of Locally Convex Spaces, p. 147-164 in "Linear Topological Spaces and Locally Complex Analysis" I, A. Aytuna (ed.), Metu-Tübitak, Ankara 1994.

[3] S.F. Bellenot, Basic sequences in non-Schwartz Fréchet spaces, Trans. Amer. Math. Soc 258 (1980), 199-216.

[4] K.D. Bierstedt and J. Bonet, Stefan Heinrich's density condition for Fréchet spaces and the characterization of distinguished Köthe echelon spaces, Math. Nachr. 135 (1988), 149-180.

[5] K.D. Bierstedt, R. Meise, W. Summers, Köthe sets and Köthe sequence spaces, p. 27-91 in "Functional Analysis, Holomorphy and Approximation Theory", North-Holland Math. Studies, 71 Amsterdam.

[6] J. Bonet, A question of Valdivia on quasinormable Fréchet spaces, Canad. Math. Bull. 34 (1991), 301-304.

[7] J. Bonet and J.C. Diaz, Distinguished subspaces and quotients of Köthe echelon spaces, Bull. Polish Acad. Sci. Math. 39 (1991), 177183.

[8] J. Bonet and J.C. Díaz, The density condition in subspaces and quotients of Fréchet spaces, Monatsh. Math. 117 (1994), 199-212.

[9] J.C. Díaz and C. Fernández, Quotients of Köthe sequence spaces of infinite onder, Arch. Math. 66 (1996), 207-213. 
[10] A. Grothendieck, Sur les espaces (F) and (DF), Summa Brasil Math. 3 (1954), 57-123.

[11] S. Heinrich, Ultrapower of locally convex spaces and applications I, Math. Nachr. 118 (1984), 211-219.

[12] H. Jarchow, Locally convex spaces, Teubner, Stuttgart, 1981.

[13] G. Köthe, Topological vector spaces I and II, Springer, BerlinHeidelberg-New York, 1969 and 1979.

[14] J. Lindenstrauss and L. Tzafriri, Classical Banach Spaces I, BerlinHeidelberg-New York, 1977.

[15] R. Meise and D. Vogt, A characterization of the quasi-normable Fréchet spaces, Math. Nachr. 122 (1985), 141-150.

[16] M. Valdivia, On quasinormable echelon space, Proc. Edinburgh Math. Soc. 24 (1981), 73-80.

[17] M. Valdivia, A characterization of totally reflexive spaces, Math. Z. 200 (1989), 327-346.

Dipartimento di Matematica "E. De Giorgi"

Università di Lecce, C.P. 193

Via per Arnesano

73100, Lecce, Italy

Recibido: 15 de Abril de 1998

Revisado: 13 de Julio de 1998 